\title{
Optimal Range of Fecal Calprotectin for Predicting Mucosal Healing in Patients with Inflammatory Bowel Disease: A Systematic Review and Meta-Analysis
}

\author{
Bing-Jie Xiang Min Jiang Ming-Jun Sun Cong Dai \\ Department of Gastroenterology, First Affiliated Hospital, China Medical University, Shenyang, China
}

\section{Keywords}

Inflammatory bowel disease · Ulcerative colitis · Fecal calprotectin - Fecal immunochemical test - Diagnostic accuracy $\cdot$ Meta-analysis

\begin{abstract}
Objective: Fecal calprotectin (FC) is a promising marker for assessment of inflammatory bowel disease (IBD) activity. However, the utility of FC for predicting mucosal healing $(\mathrm{MH})$ of IBD patients has yet to be clearly demonstrated. The objective of our study was to perform a meta-analysis evaluating the diagnostic accuracy of FC in predicting $\mathrm{MH}$ of IBD patients. Methods: We systematically searched the databases for studies from inception to April 2020 that evaluated $\mathrm{MH}$ in IBD. The methodological quality of each study was assessed according to the Quality Assessment of Diagnostic Accuracy Studies checklist. The extracted data were pooled using a summary receiver operating characteristic curve model. Random-effects model was used to summarize the diagnostic odds ratio, sensitivity, specificity, positive likelihood ratio, and negative likelihood ratio. Results: Sixteen studies comprising 1,682 ulcerative colitis (UC) patients and 4 studies comprising 221 Crohn's disease (CD) patients were included. The best performance of $\mathrm{FC}$ for predicting $\mathrm{MH}$ in UC was at cut-off range of $60-75 \mu \mathrm{g} / \mathrm{g}$ with area under the curve (AUC) of 0.88 and pooled sensitivity and specificity of 0.87 and 0.79 , respectively. The pooled sensitivity and specificity values of cutoff range $180-250 \mu \mathrm{g} / \mathrm{g}$ for predicting $\mathrm{MH}$ in CD were 0.67 and 0.76 , respectively. The AUC of 0.79 also revealed improved discrimination for identifying $\mathrm{MH}$ in $\mathrm{CD}$
\end{abstract}

with FC concentration. Conclusion: Our meta-analysis has found that $\mathrm{FC}$ is a simple, reliable noninvasive marker for predicting $\mathrm{MH}$ in IBD patients. FC cutoff range $60-75 \mu \mathrm{g} / \mathrm{g}$ appears to have the best overall accuracy in UC patients.

(c) 2021 S. Karger AG, Basel

\section{Introduction}

Inflammatory bowel disease (IBD), including ulcerative colitis (UC) and Crohn's disease (CD), have been empirically confirmed by clinical, pathological, endoscopic, and radiological features. The incidence and prevalence of IBD is increasing in worldwide [1]. The clinical course of IBD is characterized by repetitive cycles of clinical remissions and relapses. The patient's quality of life would be significantly affected during the relapse phrase. It is important to evaluate if the previous treatment is effective through clinical disease activity indices such as Mayo score, Crohn's Disease Activity Index (CDAI), and Harvey Bradshaw Index (HBI). However, the evaluation of symptom scoring for IBD patients is frequently incongruent with actual endoscopic findings. For example, Peyrin-Biroulet et al. [2] found that only 53\% of patients with clinical remission by CDAI had achieved mucosal healing $(\mathrm{MH})$. Recently, treatment of IBD is aimed at achieving $\mathrm{MH}$, because the reduced risk of relapse has been shown in IBD patients with $\mathrm{MH}$ [2]. $\mathrm{MH}$ is defined as Mayo endoscopic subscore (MES) $0 / 0-1$ or UC Endoscopic Index of Severity (UCEIS) score $0 / 0-1$ or histologic remission for UC, Simple Endoscopic Score for CD 
(SES-CD) of 0 for CD. At the same time, the targeting of $\mathrm{MH}$, rather than clinical remission, is related to improved long-term clinical outcomes for IBD, promoting the reduced risk of surgery, steroid dependence, and hospitalization, while advancing deep and sustained remission [3]. Therefore, evaluating the intestinal mucosa of IBD patients in clinical remission appears to be an important approach to tailoring therapy and predicting prognosis.

Nowadays, endoscopy remains the gold standard for assessment of intestinal inflammation of IBD [4]. However, endoscopy is not an ideal method for patients to experience repeated inspection because it is invasive, inconvenient, expensive, and related to a small but significant risk of procedural complications. Serum markers such as C-reactive protein, erythrocyte sedimentation rate, and leukocytes are used to reflect systemic inflammation of host responses. The disadvantage of these markers is not being specific for intestinal inflammation [5]. Hence, there is an urgent need for a reliable, convenient, rapid, inexpensive, reproducible, standardized noninvasive marker for MH in IBD. Fecal markers are highly accurate for detecting the mucosal inflammation and disease prognosis $[6,7]$. Besides, they are also useful for detecting the lesions in the small intestine, which could not be easily observed with endoscopy [6, 7].

Fecal markers for mucosal inflammation are a potential noninvasive alternative that could be used to evaluate $\mathrm{MH}$. The common fecal marker is fecal calprotectin (FC). FC is a cytosolic granulocyte protein associated with neutrophil migration to the intestinal tract. It is sensitive for identifying mucosal inflammation, prognosticating treatment response, and predicting disease relapse. However, the specificity of FC for $\mathrm{MH}$ has not been validated. Although the utility of FC in IBD has been evaluated in some studies, its exact role has yet to be quantified [8-12]. In this meta-analysis, we aim to evaluate the overall diagnostic accuracy of FC for predicting $\mathrm{MH}$ in IBD patients.

\section{Methods}

\section{Literature Search}

We comprehensively and systematically searched the databases including Medline (OvidSP), Web of Science, PubMed, Cochrane Library, Embase, and conference proceedings for eligible studies from inception to April 2020 that evaluated MH in IBD by FC. Language restrictions were not used. The search strategy used the following terms: (1) FC: "Fecal calprotectin", "Faecal calprotectin", "FC", "FCP"; (2) IBD: "inflammatory bowel disease", "IBD”, "ulcerative colitis", "UC", "Crohn's disease", “CD”, “colitis", “enteritis", "intestinal inflammation". References and reviews of related literature were searched manually.

\section{Study Selection}

Articles were first screened by 2 independent reviewers (Cong Dai and Bing-Jie Xiang) based on the title and abstract. The full text of a potentially eligible study was then assessed independently.

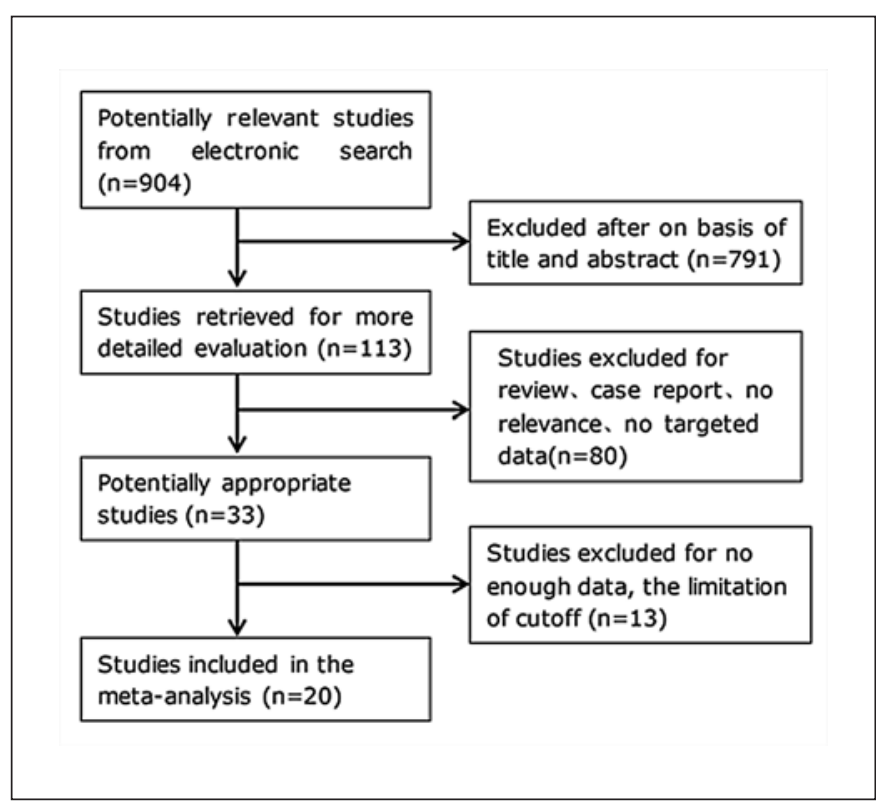

Fig. 1. Flow diagram of articles retrieved and inclusion progress through the stage of meta-analysis.

Disagreements were resolved by discussion. A study was included if it met the inclusion criteria as follows: (1) the study evaluated FC for predicting MH in IBD patients; (2) an endoscopic scoring system was used as reference standard to assess $\mathrm{MH}$; (3) the study provided sufficient details to calculate true-positive (TP), falsepositive (FP), false-negative (FN) and true-negative (TN) results.

\section{Data Extraction and Quality Assessment}

The 2 investigators (Cong Dai and Bing-Jie Xiang) who performed the database searches also independently extracted the relevant data. The retrieved data included the authors, the publication year, country, age, the reference criteria, patient characteristics, and the FC features (method, cutoff). The TP, FP, FN, and TN values were calculated for each included study.

The methodological quality of the included studies was independently assessed by 2 authors (Cong Dai and Bing-Jie Xiang) using the QUADAS-2 (Quality Assessment of Diagnostic Accuracy Studies) tool [13]. The QUADAS-2 tool comprises 4 key domains: patient selection, index test, reference standard, and flow and timing. Each domain is assessed in terms of the risk of bias, and the first 3 domains are additionally assessed in terms of concerns regarding applicability [14]. In any case of disagreement, a consensus was reached by consultation with the senior reviewer (Ming-Jun Sun).

\section{Data Synthesis and Statistical Analysis}

The standard methods were used in this meta-analysis as recommended in the Cochrane handbook for systematic reviews of diagnostic test accuracy. For each study, sensitivity, specificity, the positive likelihood ratio (PLR), the negative likelihood ratio (NLR), and the diagnostic odds ratio (DOR) were calculated. The DOR combines both the PLRs and NLRs and is a global measure of test performance. A higher DOR value represents better discriminatory performance. For the data analysis, we estimated both summary receiver operating characteristic (SROC) curves and average operating points with each commonly applied threshold value, as they may complement each other in providing clinically useful summaries and powerful ways of detecting effects. An SROC curve 
Table 1. Characteristics of included studies

\begin{tabular}{|c|c|c|c|c|c|c|c|c|c|c|c|}
\hline Study & Year & Country & Age, years & Type & Criteria & $\mathrm{FC}, \mu \mathrm{g} / \mathrm{g}$ & Method & ТP & FP & FN & $\mathrm{TN}$ \\
\hline Carlsen et al. [30] & 2018 & Multicenter & $44(29-52)$ & Prospective & MES 0 & 25 & ELISA & 10 & 6 & 15 & 64 \\
\hline Goll et al. [24] & 2019 & Norway & NA & Prospective & MES 0 & 28 & ELISA & 16 & 11 & 26 & 51 \\
\hline Theede et al. [25] & 2016 & Denmark & $39.3(13.92)$ & Prospective & HS 0 & 45 & ELISA & 28 & 0 & 33 & 8 \\
\hline Sandborn et al. [28] & 2016 & Multicenter & $39.9 \pm 15.1$ & Prospective & MES 0 & 50 & ELISA & 29 & 77 & 4 & 84 \\
\hline Patel et al. [16] & 2017 & USA & $26(2-56)$ & Retrospective & PRO2 $0+$ MES 0 & 60 & ELISA & 12 & 2 & 2 & 52 \\
\hline Kristensen et al. [22] & 2015 & Norway & $35.5(18-72)$ & Prospective & MES 0 & 61 & ELISA & 37 & 3 & 7 & 15 \\
\hline Kim et al. [20] & 2020 & Korea & $50(18-81)$ & Prospective & MES 0 & 70 & ELISA & 58 & 18 & 7 & 44 \\
\hline Carlsen et al. [30] & 2018 & Multicenter & $44(29-52)$ & Prospective & MES $0+$ Geboes 0/1 & 74 & ELISA & 11 & 26 & 1 & 51 \\
\hline Sandborn et al. [28] & 2016 & Multicenter & $39.9 \pm 15.1$ & Prospective & MES 0 & 75 & ELISA & 28 & 53 & 5 & 108 \\
\hline Kristensen et al. [22] & 2015 & Norway & $35.5(18-72)$ & Prospective & MES 0 & 96 & ELISA & 40 & 3 & 4 & 15 \\
\hline Kim et al. [20] & 2020 & Korea & $50(18-81)$ & Prospective & MES 0 & 100 & ELISA & 58 & 21 & 7 & 41 \\
\hline Sandborn et al. [28] & 2016 & Multicenter & $39.9 \pm 15.1$ & Prospective & MES 0 & 100 & ELISA & 27 & 53 & 6 & 108 \\
\hline Goll et al. [24] & 2019 & Norway & NA & Prospective & MES 0 & 100 & ELISA & 36 & 25 & 6 & 37 \\
\hline Lee et al. [19] & 2019 & Korea & $48.4 \pm 13.2(23-80)$ & Prospective & MES 0 & 150 & QPOCT & 6 & 0 & 1 & 22 \\
\hline Carlsen et al. [30] & 2018 & Multicenter & $44(29-52)$ & Prospective & MES $0+$ Geboes $0 / 1$ & 150 & ELISA & 11 & 36 & 1 & 41 \\
\hline Sandborn et al. [28] & 2016 & Multicenter & $39.9 \pm 15.1$ & Prospective & MES 0 & 150 & ELISA & 26 & 41 & 7 & 120 \\
\hline Ryu et al. [17] & 2019 & Korea & $47.2(16-78)$ & Retrospective & MES 0 & 170 & ELISA & 40 & 31 & 11 & 92 \\
\hline Hiraoka et al. [11] & 2018 & Japan & $32(22-43)$ & Prospective & MES 0 & 180 & ELISA & 84 & 24 & 38 & 75 \\
\hline Theede et al. [15] & 2015 & Denmark & $19-79$ & Retrospective & MES 0 & 192 & ELISA & 24 & 10 & 8 & 78 \\
\hline Yamaguchi et al. [29] & 2016 & Multicenter & $45(36-54)$ & Prospective & MES 0 & 194 & ELISA & 37 & 22 & 15 & 31 \\
\hline Takashima et al. [8] & 2015 & Japan & $35.5(14-77)$ & Prospective & MES 0 & 200 & ELISA & 34 & 17 & 10 & 44 \\
\hline Sandborn et al. [28] & 2016 & Multicenter & $39.9 \pm 15.1$ & Prospective & MES 0 & 200 & ELISA & 23 & 29 & 10 & 132 \\
\hline Mak et al. [26] & 2018 & USA & $36.0 \pm 18.7$ & Prospective & MES 0 & 200 & ELISA & 4 & 11 & 1 & 45 \\
\hline Lee et al. [19] & 2019 & Korea & $48.4 \pm 13.2(23-80)$ & Prospective & MES 0 & 201 & ELISA & 5 & 0 & 2 & 22 \\
\hline Ryu et al. [17] & 2019 & Korea & $47.2(16-78)$ & Retrospective & UCEIS $0 / 1$ & 170 & ELISA & 44 & 27 & 15 & 98 \\
\hline Sandborn et al. [28] & 2016 & Multicenter & $39.9 \pm 15.1$ & Prospective & MES 0-1 & 200 & ELISA & 26 & 69 & 7 & 92 \\
\hline Yamaguchi et al. [29] & 2016 & Multicenter & $45(36-54)$ & Prospective & MES 0-1 & 200 & ELISA & 63 & 1 & 31 & 10 \\
\hline Mak et al. [26] & 2018 & USA & $36.0 \pm 18.7$ & Prospective & HS & 200 & ELISA & 5 & 13 & 2 & 41 \\
\hline Takashima et al. [8] & 2015 & Japan & $35.5(14-77)$ & Prospective & MES 0-1 & 250 & ELISA & 45 & 14 & 19 & 27 \\
\hline Kristensen et al. [23] & 2017 & Norway & $31(18-60)$ & Prospective & MES 0-1 & 250 & ELISA & 16 & 0 & 3 & 1 \\
\hline Sandborn et al. [28] & 2016 & Multicenter & $39.9 \pm 15.1$ & Prospective & MES 0-1 & 250 & ELISA & 25 & 68 & 8 & 93 \\
\hline D'Haens et al. [21] & 2012 & Belgium & $38(30-46)$ & Prospective & MES 0/1 & 250 & ELISA & 14 & 3 & 4 & 18 \\
\hline Mak et al. [26] & 2018 & USA & $36.0 \pm 18.7$ & Prospective & MES 0-1 & 250 & ELISA & 3 & 13 & 2 & 43 \\
\hline Kristensen et al. [22] & 2015 & Norway & $35.5(18-72)$ & Prospective & MES 0-1 & 259 & ELISA & 25 & 9 & 5 & 23 \\
\hline Arai et al. [18] & 2017 & Japan & $31.8(15-69)$ & Prospective & SES-CD 0 & 180 & ELISA & 4 & 1 & 6 & 13 \\
\hline Inokuchi et al. [9] & 2016 & Japan & $32(22-43)$ & Prospective & SES-CD 0 & 180 & ELISA & 20 & 14 & 3 & 34 \\
\hline Ma et al. [10] & 2017 & Canada & $47.9(22-75)$ & Prospective & SES-CD 0 & 215 & ELISA & 36 & 12 & 7 & 31 \\
\hline Zubin and Peter [27] & 2015 & Australia & $13.5(12.2-13.88)$ & Prospective & SES-CD 0 & 250 & ELISA & 11 & 4 & 15 & 10 \\
\hline
\end{tabular}

QPOCT, quantitative point-of-care test; ELISA, enzyme-linked immunosorbent assay; PRO2, patient-reported outcome measures; HS, histologic inflammatory activity score.

with $95 \%$ confidence region and $95 \%$ prediction region was performed to examine the interaction between sensitivity and specificity. DOR and the area under the SROC curve (AUC) were calculated to evaluate the diagnostic performance of $\mathrm{FC}$ for $\mathrm{MH}$ in IBD patients. A DOR of 1 indicates that the test cannot discriminate between patients with $\mathrm{MH}$ and non-MH UC patients. A higher value indicates better test performance. AUC equals 1 for a perfect test and 0.5 for a completely uninformative test. When AUC was $0.5-0.7$, it revealed there was a certain diagnostic efficacy. AUC $>0.8$ signified that diagnostic efficacy was outstanding, and AUC $>0.9$ signified that diagnostic efficacy was significantly outstanding. Pooled sensitivity, specificity, and their corresponding 95\% CIs were calculated using a random effects model at each threshold. The heterogeneity was detected by a chi-square test or Q-statistic and Higgins I-squared statistic $\left(I^{2}\right)$. A $p$ value of $<0.1$ was considered statistically significant heterogeneity for the chisquare or Q-statistics. The percentage of $I^{2}$ represented the degree of heterogeneity. $I^{2}$ percentages of 25,50 , and $75 \%$ indicated a low, moderate, and high degree of heterogeneity, respectively. We resolved the heterogeneity by sensitivity analysis. Publication bias was assessed using Deeks' test. $p<0.05$ was considered to indicate statistically significant publication bias. We performed statistical analysis on Stata (version 14) and Review Manager (version 5.3).

\section{Results}

\section{Study Characteristics}

As Figure 1 shows, 904 articles are available after the initial search. After reading the titles and abstracts and reviewing the full texts, 16 publications including 1,682 UC patients and 4 publications including $221 \mathrm{CD}$ patients were included in the final analysis. The clinical characteristics of the included studies are listed in Table 1. All stud- 


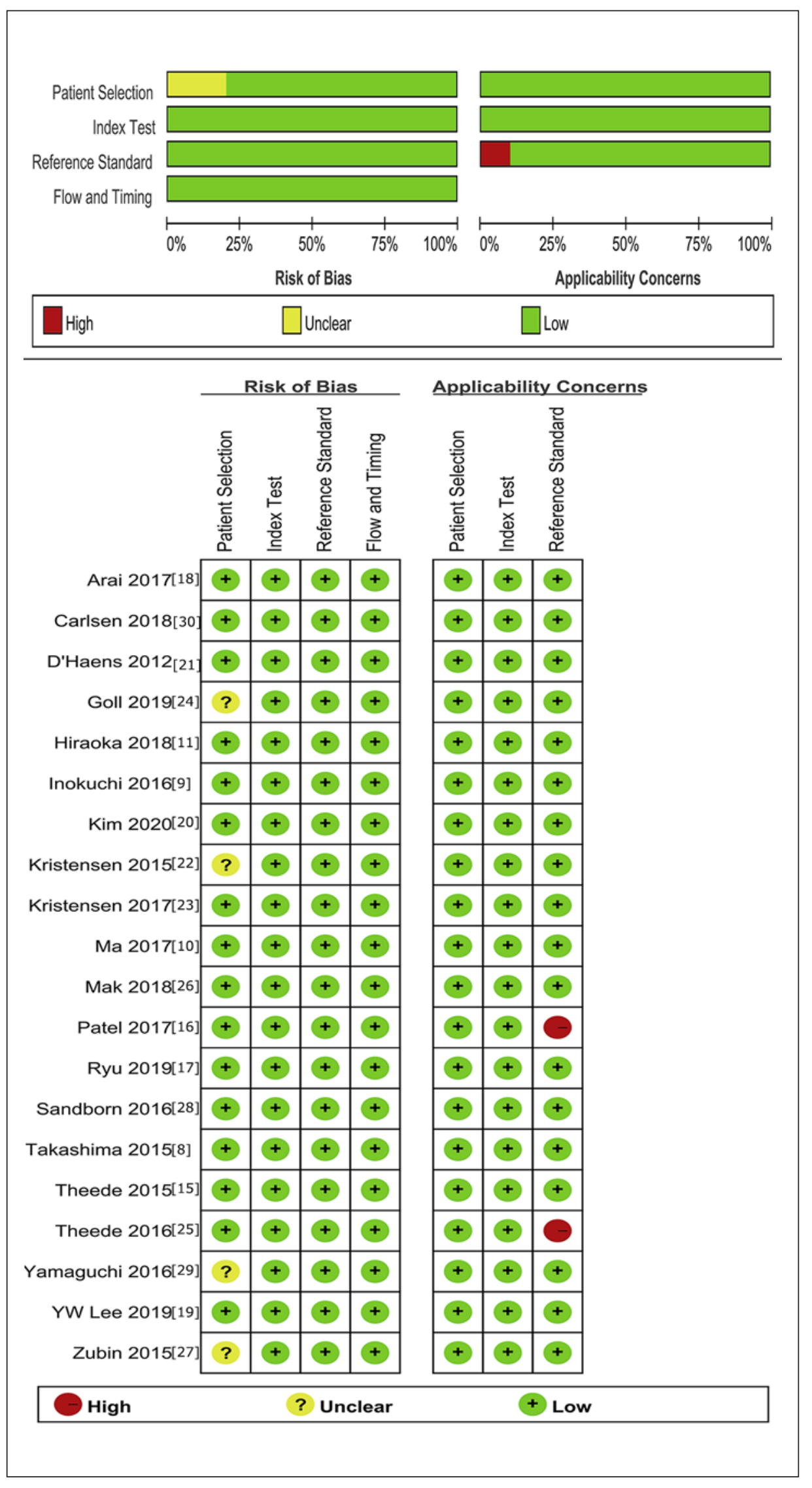

Fig. 2. Methodological quality graph and summary of the included studies based on the Cochrane handbook. +, low risk; -, high risk; ?, unclear. 


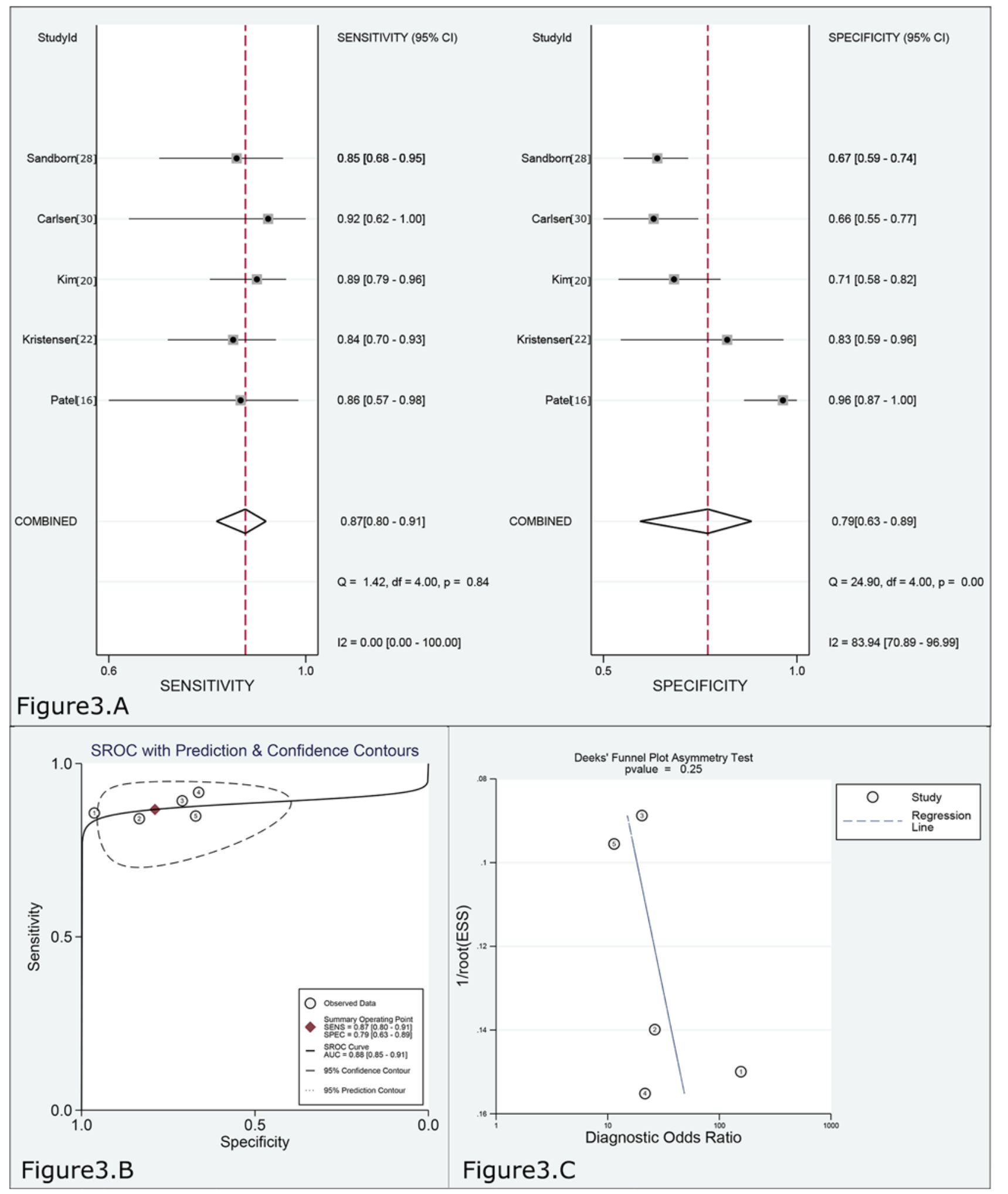

Fig. 3. A Forest plot of the sensitivity and specificity for predicting $\mathrm{MH}$ in UC by FC. B SROC curve for predicting $\mathrm{MH}$ in UC by FC. C Deeks' funnel plot for evaluating publication bias (cut-off range $=60-75 \mu \mathrm{g} / \mathrm{g}$ ). 
Table 2. Summary of analysis results

\begin{tabular}{llllrlrrr}
\hline Criteria & FC & Sensitivity & Specificity & DOR & PLR & NLR & AUC & $I^{2}, \%$ \\
\hline MES 0, HS 0 & $25-50$ & 0.54 & 0.81 & 5 & 2.9 & 0.56 & 0.76 & 96 \\
MES 0 & $60-75$ & 0.87 & 0.79 & 24 & 4.1 & 0.17 & 0.88 & 59 \\
MES 0 & $60-75^{*}$ & 0.88 & 0.78 & 25 & 4.0 & 0.16 & 0.89 & 0 \\
MES 0 & $96-125$ & 0.87 & 0.66 & 14 & 2.6 & 0.19 & 0.86 & 0 \\
MES 0 & $150-180$ & 0.75 & 0.75 & 9 & 3.0 & 0.33 & 0.81 & 60 \\
MES 0 & $150-180^{*}$ & 0.80 & 0.78 & 15 & 3.7 & 0.25 & 0.82 & 0 \\
MES 0 & $192-201$ & 0.74 & 0.82 & 12 & 4.0 & 0.32 & 0.75 & 70 \\
MES 0 & $192-201^{*}$ & 0.76 & 0.82 & 13 & 4.0 & 0.31 & 0.79 & 70 \\
MES/UCEIS 0-1/HS & $170-200$ & 0.73 & 0.74 & 8 & 2.8 & 0.37 & 0.78 & 68 \\
MES 0-1 & $250-259$ & 0.76 & 0.70 & 8 & 2.6 & 0.34 & 0.79 & 0 \\
SES-CD 0 & $180-250$ & 0.67 & 0.76 & 7 & 2.9 & 0.43 & 0.79 & 79 \\
\hline
\end{tabular}

* Sensitivity analysis. MES, Mayo Endoscopic Subscore; HS, histologic inflammatory activity score.

ies enrolled patients with diagnosed UC or CD. Most studies except 3 studies [15-17] used a prospective study design. Seven of the studies were conducted in Asia (4 studies in Japan $[8,9,11,18], 3$ studies in Korea [17, 19, 20]), 6 of the studies were in Europe (1 in Belgium [21], 3 in Norway [22-24], 2 in Denmark [15, 25]), 1 of the studies was in Canada [10], 2 of the studies were in the USA $[16,26], 1$ of the studies was in Australia [27], and 3 of the studies were multicenter [28-30]. Reference standards of most included studies were based on endoscopy, including MES, UCEIS in MH evaluation of UC, and SES-CD in $\mathrm{MH}$ evaluation of $\mathrm{CD}$. Reference standard of 2 studies $[25,26]$ was based on histologic inflammatory activity. Taking limitation of study number, cut-off of endoscopic score, and cut-off of FC into consideration, we evaluated 7 FC level cut-off ranges in UC as per availability of reports in the included studies: $25-50,60-75,96-125,150$ $180,192-201,170-200,250-259 \mu \mathrm{g} / \mathrm{g}$. We evaluated $1 \mathrm{FC}$ level cut-off range $(180-250 \mu \mathrm{g} / \mathrm{g})$ in CD as per availability of reports in the included studies. When $\mathrm{MH}$ was defined as MES 0, 4 studies [15, 24, 28, 30] (MH was based on histologic activity evaluation in 1 study [25]) about FC with cut-off range of $25-50 \mu \mathrm{g} / \mathrm{g}$, 5 studies [16, 20, 22, 28, 30 ] about FC with cut-off range of $60-75 \mu \mathrm{g} / \mathrm{g}, 4$ studies $[20,22,24,28]$ about FC with cut-off range of 96-125 $\mu \mathrm{g} / \mathrm{g}, 5$ studies $[11,17,19,28,30]$ about FC with cutoff range of $150-180 \mu \mathrm{g} / \mathrm{g}$, and 6 studies $[8,15,19,26,28,29]$ about FC with cut-off range of $192-201 \mu \mathrm{g} / \mathrm{g}$ for predicting $\mathrm{MH}$ in UC patients were included in the final metaanalysis (Table 1). When MH was defined as MES $0-1$ or UCEIS $0-1,4$ studies [17, 26, 28, 29] (MH was based on histologic activity evaluation in 1 study [26]) about FC with cut-off range of $170-200 \mu \mathrm{g} / \mathrm{g}$ and 6 studies $[8,21-$ $23,26,28]$ about FC with cut-off range of $250-259 \mu \mathrm{g} / \mathrm{g}$ for predicting $\mathrm{MH}$ in UC patients were included in the final meta-analysis (Table 1). When $\mathrm{MH}$ was defined as
SES-CD 0, 4 studies $[9,10,18,27]$ about FC with cut-off range of $180-250 \mu \mathrm{g} / \mathrm{g}$ for predicting $\mathrm{MH}$ in CD patients were included in the final meta-analysis (Table 1).

\section{Methodological Quality Assessment}

The 20 studies underwent quality assessment using the aforementioned QUADAS-2 tool. A summary of the results is presented in Figure 2. Most studies were deemed to have a representative spectrum of patients. The criteria for both $\mathrm{MH}$ were present in Table 1 . Specifically, 2 studies $[25,26]$ defined $\mathrm{MH}$ in $\mathrm{UC}$ as histologic remission, and 1 study [16] defined MH in UC as MES 0 and patientreported outcome measures (PRO2) 0. One study [30] defined MH in UC as MES 0 and Geboes 0/1. There was no evidence of commercial funding in any of the studies; however, not all of the manuscripts explicitly verified this with a funding statement.

\section{Diagnostic Accuracy Meta-Analyses}

The pooled sensitivity and specificity values of $60-75$ $\mu \mathrm{g} / \mathrm{g}$ for predicting MES 0 in UC were 0.87 (95\% CI 0.80 $0.91,5$ studies, $n=540)$ and 0.79 (95\% CI 0.63-0.89), respectively (Fig. 3A, Table 2). The FC level had a high rulein value (PLR 4.1; 95\% CI 2.2-7.5) and a low rule-out value (NLR 0.17; 95\% CI 0.11-0.25) for predicting $\mathrm{MH}$ in UC. The results of the ROC curve analysis (AUC 0.88, 95\% CI 0.85-0.91; Fig. 3B, Table 2$)$ and DOR (24, 95\% CI 11-56) also revealed outstanding discrimination for predicting $\mathrm{MH}$ in UC with FC concentration. $I^{2}$ was $59 \%$ $(p=0.043)$; thus, the sensitivity analysis was performed after excluding 1 study and obtained the following results (online suppl. Fig. 2; for all online suppl. material, see www.karger.com/doi/10.1159/000514196). The pooled sensitivity and specificity were 0.88 (95\% CI $0.80-0.93$ ) and 0.78 (95\% CI 0.59-0.90), respectively. PLR and NLR were 4.0 (95\% CI 2.0-8.1) and 0.16 (95\% CI 0.09-0.26), 


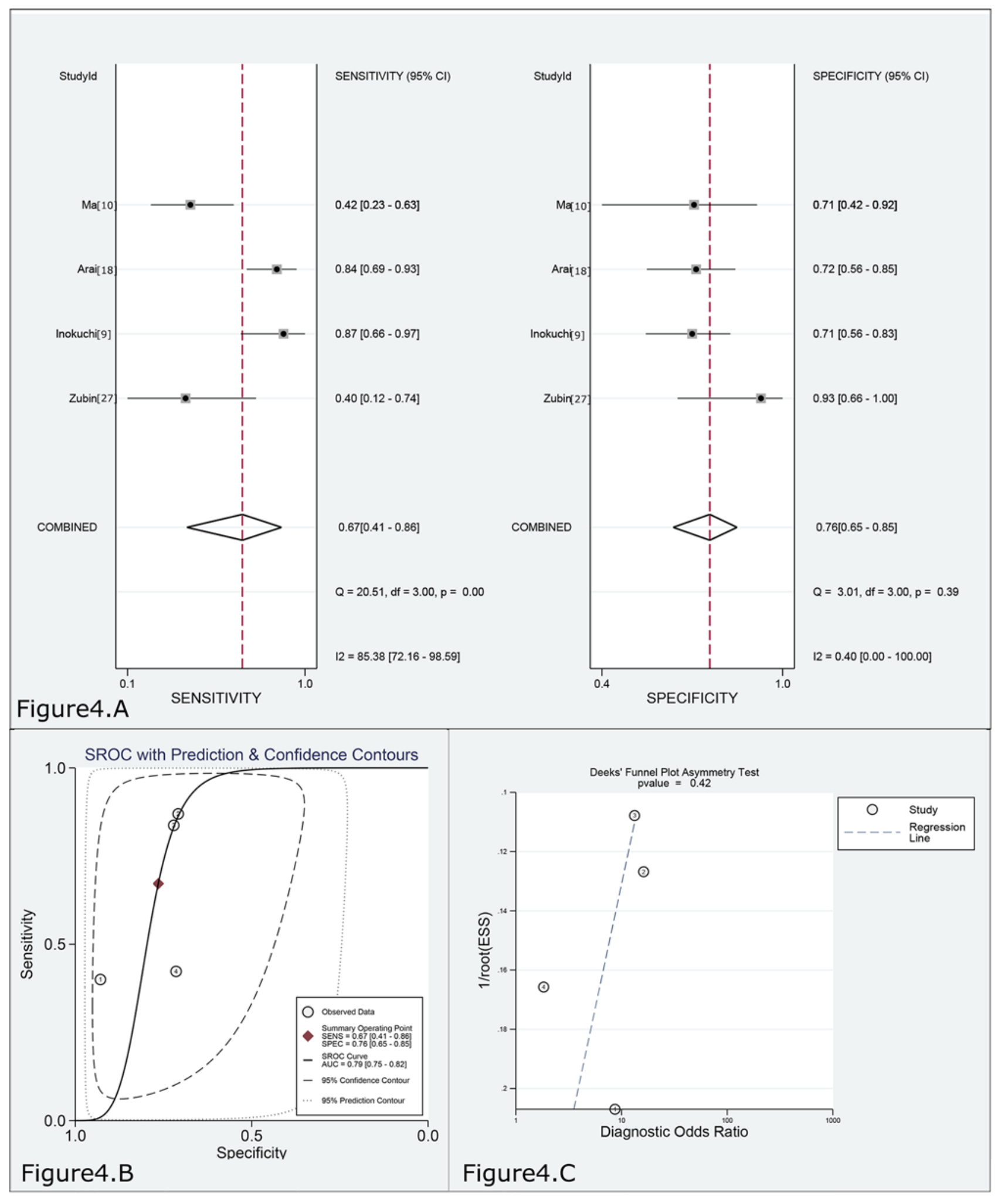

Fig. 4. A Forest plot of the sensitivity and specificity for predicting $\mathrm{MH}$ in $\mathrm{CD}$ by FC. B SROC curve for predicting MH in CD by FC. C Deeks' funnel plot for evaluating publication bias (cut-off range $=180-250 \mu \mathrm{g} / \mathrm{g}$ ). 
respectively. AUC and DOR were 0.89 (95\% CI $0.86-$ 0.91 ) and 25 (95\% CI 9-71), respectively.

The diagnostic performances of other values including $25-50 \mu \mathrm{g} / \mathrm{g}, 96-125 \mu \mathrm{g} / \mathrm{g}, 150-180 \mu \mathrm{g} / \mathrm{g}, 192-201 \mu \mathrm{g} / \mathrm{g}$, $170-200 \mu \mathrm{g} / \mathrm{g}$, and $250-259 \mu \mathrm{g} / \mathrm{g}$ in predicting $\mathrm{MH}$ of UC are presented in Table 2 and online supplementary Figures 1, 3-9.

The pooled sensitivity and specificity values of 180 $250 \mu \mathrm{g} / \mathrm{g}$ for predicting SES-CD 0 in CD were 0.67 (95\% CI $0.41-0.86$, 4 studies, $n=221)$ and 0.76 (95\% CI $0.65-$ 0.85 ), respectively (Fig. 4A, Table 2). The FC level had a moderate rule-in value (PLR 2.9; 95\% CI 1.8-4.4) and a moderate rule-out value (NLR 0.43 ; 95\% CI $0.22-0.86$ ) for predicting $\mathrm{MH}$ in $\mathrm{CD}$. The results of the ROC curve analysis (AUC $0.79,95 \%$ CI 0.75-0.82; Fig. 4B, Table 2) and DOR (7, 95\% CI 2-18) revealed diagnostic discrimination for predicting $\mathrm{MH}$ in $\mathrm{CD}$ with $\mathrm{FC}$ concentration. $I^{2}$ was $79 \%(p<0.01)$, but the sensitivity analysis could not be performed due to quantitative limitation of included studies.

\section{Publication Bias}

Funnel plots for the analyses of publication bias were performed to compare the yield of FC levels for assessing $\mathrm{MH}$ in UC or CD. The Deeks' tests revealed no evidence of publication bias in all analyses $(p=0.19-0.77$; online suppl. Figs. 3-10).

\section{Discussion}

To our knowledge, the present study was the first meta-analysis of assessment of $\mathrm{MH}$ in IBD patients using an FC assay. In this study, through a robust systematic review and an appropriately performed meta-analysis, FC has the best performance for predicting MH in UC at cutoff range of $60-75 \mu \mathrm{g} / \mathrm{g}$, achieving a high sensitivity $(0.87$, 95\% CI $0.80-0.91)$ and high specificity $(0.81,95 \%$ CI 0.76-0.85). The estimated DOR for the FC in predicting $\mathrm{MH}$ of UC was 24 in our meta-analysis. This means that for the FC, the odds for positivity among subjects with $\mathrm{MH}$ of UC patients is 24 times higher than the odds for positivity among subjects without $\mathrm{MH}$ of UC patients. Compared with DOR, likelihood ratios including PLR and NLR are considered more clinically useful. In our meta-analysis, the pooled PLR and NLR were 4.10 and 0.17 , respectively, suggesting that UC patients with $\mathrm{MH}$ are 4 -fold more likely to have lower FC levels. In contrast, if the FC level of the UC patients is above the cut-off value, the probability of non- $\mathrm{MH}$ is $17 \%$.

FC has a moderate sensitivity $(0.67,95 \%$ CI $0.41-0.86)$ and a high specificity $(0.76,95 \%$ CI $0.65-0.85)$ for predicting $\mathrm{MH}$ in $\mathrm{CD}$. The estimated DOR for the FC in predicting $\mathrm{MH}$ of $\mathrm{CD}$ was 7 in our meta-analysis. This means that for the FC, the odds for positivity among subjects with $\mathrm{MH}$ of $\mathrm{CD}$ patients is 7 times higher than the odds for positivity among subjects without $\mathrm{MH}$ of $\mathrm{CD}$ patients. Compared with DOR, likelihood ratios including PLR and NLR are considered more clinically useful. In our meta-analysis, the pooled PLR and NLR were 2.9 and 0.43 , respectively, suggesting that CD patients with $\mathrm{MH}$ are approximately 3-fold more likely to have lower FC levels. In contrast, if the FC level of the CD patients is above the cutoff value, the probability of non-MH is $43 \%$.

The common fecal markers in IBD include FC, fecal lactoferrin (FL) and fecal immunochemical test (FIT). FIT measures the amount of blood from the lesion in intestinal tract, whereas the fundamental of FC and FL estimating inflammation activity in intestinal tract is associated with the amount of inflammatory cells [31]. Calprotectin, a calcium and zinc-binding protein of 36.5 $\mathrm{kDa}$, represents $60 \%$ of cytosolic proteins in the granulocytes and it appears on the surface of granulocytes [32, 33]. The concentration of calprotectin in stools is therefore correlated with the amount of neutrophil infiltrating in the inflamed mucosa of IBD [33, 34]. Lactoferrin is a $76-\mathrm{kDa}$ transferrin with high affinity to iron ions. It is excreted by the majority of mucosal membranes and isolated from various secretions, such as saliva, breast milk, tears, nasal secretions, stool, and serum [35-37]. Polymorphonuclear neutrophils migrate to the mucosa during the inflammatory process of gastrointestinal tract, which promotes the increase of lactoferrin concentration in feces [38]. In distinction to FC, the use of FL testing has been mainly limited to research, probably because stability of lactoferrin is shorter than calprotectin at room temperature. FIT is a technique based on measurement of gut hemoglobin, not specific for intestinal inflammation. Thus, the elevation of FIT levels will results from any cause of increased red blood cells in bowel. Previous studies have shown that FIT is helpful for identifying $\mathrm{MH}$ in UC $[11,12]$. However, the present study showed the performance of FC at cut-off range of $60-75 \mu \mathrm{g} / \mathrm{g}$ for predicting $\mathrm{MH}$ was better than that of FIT in previous metaanalysis [39], in which FIT had a pooled sensitivity, specificity, and DOR of $0.77,0.81$, and 18.8 for predicting $\mathrm{MH}$ in UC. Given a comparable accuracy to FIT and FL, FC has potential to become a promising tool for predicting $\mathrm{MH}$ in IBD patients.

FC has some disadvantages in predicting $\mathrm{MH}$ for IBD. First, dietary supplements such as vitamin D, fatty acids, zinc, environmental influences (intestinal bacterial flora), and genetic influences can affect FC levels. Second, FC is not generally obtainable in most countries due to higher expense and non-reimbursement compared with FIT. FIT assessment costs USD 26 in China, whereas FC approximately costs USD 200 [31]. Third, FC is analyzed by 
ELISA and the measurement requires 5-10 $\mathrm{g}$ of fecal material [40] and specific skills. Besides, the process takes several hours to perform. The stool processing steps also take a lot of time. Nowadays, 2 types of point-of-care tests have been developed: semi-quantitative tests that provide a range of FC levels and a rapid quantitative point-of-care test (QPOCT), which is as fast as the semi-quantitative tests but provides an exact number as the ELISA test [41, 42]. A good correlation between QPOCT and ELISA test was observed in previous studies, which also explored the ability of QPOCT to predict endoscopic activity in IBD patients [19, 43, 44]. Recently, Vinding et al. [45] have validated a home-based rapid test in the laboratory, but not in the hand of patients. Elkjaer et al. [46] have developed of a rapid home test by CALPRO, Inc., Oslo, Norway, and evaluated its ability in their laboratory. CalproSmart, a further development of the rapid home test, is performed by the patients themselves and can get the result within minutes. The fast and reliable tool allows for evaluation of gut inflammation by the patient themselves and the results thereby constitute a valuable addition to clinical evidence. But its reliability can be affected by educational level, which signifies that thorough instruction, guidance, and follow-up are needed if the tool is implemented in clinical practice. But these FC tests are only carried out in the laboratory, and future trials are warranted to verify these value in clinical.

This meta-analysis successfully determined the optimum cut-off range of FC for identifying $\mathrm{MH}$ in $\mathrm{UC}$ and confirmed the diagnostic accuracy of FC for identifying $\mathrm{MH}$ in CD. However, this meta-analysis has several limitations. First, most of the included studies were carried out in tertiary centers. Thus, the study was limited to IBD population that were enrolled in the primary cares. The usage of FC test was limited due to this spectrum bias in primary cares with a lower activity probability. More multicenter large-sample randomized controlled trials (RCTs) and strict patient access systems are required to precisely investigate the predictive performance of FC in IBD patients. Second, although the endoscopic evaluation was used as reference standard, there is still no consensus between endoscopic scoring systems for UC. For example, one study defined MH using UCEIS [17], whereas other studies defined MH using MES $[28,29]$. Moreover, the definition of $\mathrm{MH}$ was based on histologic evaluation in 2 included studies [25, 26]. And definition of $\mathrm{MH}$ involved clinical activity or histologic activity in 2 studies $[16,30]$. Third, the standardized measurement methods of FC have not been established. Various assay kits such as Calprest [24] and Calprotectin [22] ELISA were used to examine FC in IBD patients in these studies. Fourth, further information about clinical characteristics of patients is unavailable, which hindered us from investigating sources of heterogeneity by disease severity (mild, moderate, or severe disease) and disease location (distal or proximal involvement). For example, CD could not be classified into distal colonic, terminal ileal, or small intestinal group in this study due to insufficient data. It is hoped that more widespread multicenter large samples and implementation of the Standards for the Reporting of Diagnostic Accuracy studies will enable readers to directly extract desired information for assessment.

In conclusion, our study revealed that FC is a simple, reliable noninvasive marker for predicting $\mathrm{MH}$ in IBD patients, especially in UC patients, and the best performance of FC cutoff range for predicting $\mathrm{MH}$ in $\mathrm{UC}$ was 60-75 $\mu \mathrm{g} / \mathrm{g}$. Further appropriately designed studies (especially RCTs) are required to verify such benefits and to find the best strategy of FC to predict $\mathrm{MH}$ in IBD patients.

\section{Acknowledgement}

All authors were involved in the conception and design of the study, acquisition of data, data analysis, and the drafting, revision, and approval of the final version of the manuscript.

\section{Statement of Ethics}

The paper is exempt from ethical committee approval because this study is a meta-analysis.

\section{Conflict of Interest Statement}

The authors declare that they have no competing interests.

\section{Funding Sources}

This research was supported by Liaoning Science and Technology Foundation (No. 20170541052).

\section{Author Contributions}

C.D. and B.-J.X. carried out the literature search, selection, validity assessment, data abstraction, and data analysis. C.D., B.-J.X., and M.J. wrote the paper and incorporated the comments from other authors and peer reviewers. C.D. and M.-J.S. had the original idea for the paper, formulated the protocol, and contributed to data abstraction and analysis. All authors reviewed and approved the final draft of the paper. 


\section{References}

1 Ng SC, Shi HY, Hamidi N, Underwood FE, Tang W, Benchimol EI, et al. Worldwide incidence and prevalence of inflammatory bowel disease in the 21st century: a systematic review of population-based studies. Lancet. 2018 Dec;390(10114):2769-78.

2 Peyrin-Biroulet L, Ferrante M, Magro F, Campbell S, Franchimont D, Fidder H, et al.; Scientific Committee of the European Crohn's and Colitis Organization. Results from the 2nd Scientific Workshop of the ECCO. I: impact of mucosal healing on the course of inflammatory bowel disease. J Crohn's Colitis. 2011 Oct;5(5):477-83.

3 Reinink AR, Lee TC, Higgins PD. Endoscopic Mucosal Healing Predicts Favorable Clinical Outcomes in Inflammatory Bowel Disease: A Meta-analysis. Inflamm Bowel Dis. 2016 Aug; 22(8):1859-69.

4 Oliva S, Di Nardo G, Hassan C, Spada C, Aloi $\mathrm{M}$, Ferrari F, et al. Second-generation colon capsule endoscopy vs. colonoscopy in pediatric ulcerative colitis: a pilot study. Endoscopy. 2014 Jun;46(6):485-92.

5 Krzystek-Korpacka M, Neubauer K, Berdowska I, Boehm D, Zielinski B, Petryszyn P, et al. Enhanced formation of advanced oxidation protein products in IBD. Inflamm Bowel Dis. 2008 Jun;14(6):794-802.

6 Judd TA, Day AS, Lemberg DA, Turner D, Leach ST. Update of fecal markers of inflammation in inflammatory bowel disease. J Gastroenterol Hepatol. 2011 Oct;26(10):1493-9.

7 Wright EK, Kamm MA, De Cruz P, Hamilton AL, Ritchie KJ, Keenan JI, et al. Comparison of Fecal Inflammatory Markers in Crohn's Disease. Inflamm Bowel Dis. 2016 May;22(5): 1086-94.

8 Takashima S, Kato J, Hiraoka S, Nakarai A, Takei D, Inokuchi $\mathrm{T}$, et al. Evaluation of $\mathrm{Mu}$ cosal Healing in Ulcerative Colitis by Fecal Calprotectin Vs. Fecal Immunochemical Test. Am J Gastroenterol. 2015 Jun;110(6): 873-80.

9 Inokuchi T, Kato J, Hiraoka S, Takashima S, Nakarai A, Takei D, et al. Fecal Immunochemical Test Versus Fecal Calprotectin for Prediction of Mucosal Healing in Crohn's Disease. Inflamm Bowel Dis. 2016 May;22(5): 1078-85.

10 Ma C, Lumb R, Walker EV, Foshaug RR, Dang TT, Verma S, et al. Noninvasive Fecal Immunochemical Testing and Fecal Calprotectin Predict Mucosal Healing in Inflammatory Bowel Disease: A Prospective Cohort Study. Inflamm Bowel Dis. 2017 Sep;23(9): 1643-9.

11 Hiraoka S, Inokuchi T, Nakarai A, Takashima S, Takei D, Sugihara Y, et al. Fecal Immunochemical Test and Fecal Calprotectin Results Show Different Profiles in Disease Monitoring for Ulcerative Colitis. Gut Liver. 2018 Mar;12(2):142-8.

12 Nakarai A, Hiraoka S, Takahashi S, Inaba T, Higashi R, Mizuno M, et al. Simultaneous Measurements of Faecal Calprotectin and the Faecal Immunochemical Test in Quiescent Ulcerative Colitis Patients Can Stratify Risk of Relapse. J Crohn's Colitis. 2018 Jan;12(1):716.
13 Whiting PF, Rutjes AW, Westwood ME, Mallett S, Deeks JJ, Reitsma JB, et al.; QUADAS-2 Group. QUADAS-2: a revised tool for the quality assessment of diagnostic accuracy studies. Ann Intern Med. 2011 Oct;155(8): 529-36.

14 Lundh A, Gøtzsche PC. Recommendations by Cochrane Review Groups for assessment of the risk of bias in studies. BMC Med Res Methodol. 2008 Apr;8(1):22.

15 Theede K, Holck S, Ibsen P, Ladelund S, Nordgaard-Lassen I, Nielsen AM. Level of Fecal Calprotectin Correlates With Endoscopic and Histologic Inflammation and Identifies $\mathrm{Pa}$ tients With Mucosal Healing in Ulcerative Colitis. Clin Gastroenterol Hepatol. 2015 Nov;13(11):1929-36.e1.

16 Patel A, Panchal H, Dubinsky MC. Fecal Calprotectin Levels Predict Histological Healing in Ulcerative Colitis. Inflamm Bowel Dis. 2017 Sep;23(9):1600-4.

17 Ryu DG, Kim HW, Park SB, Kang DH, Choi $\mathrm{CW}, \mathrm{Kim}$ SJ, et al. Clinical implications of fecal calprotectin and fecal immunochemical test on mucosal status in patients with ulcerative colitis. Medicine (Baltimore). 2019 Sep; 98(36):e17080

18 Arai T, Takeuchi K, Miyamura M, Ishikawa R, Yamada A, Katsumata M, et al. Level of Fecal Calprotectin Correlates With Severity of Small Bowel Crohn's Disease, Measured by Balloon-assisted Enteroscopy and Computed Tomography Enterography. Clin Gastroenterol Hepatol. 2017 Jan;15(1):56-62.

19 Lee YW, Lee KM, Lee JM, Chung YY, Kim DB, Kim YJ, et al. The usefulness of fecal calprotectin in assessing inflammatory bowel disease activity. Korean J Intern Med (Korean Assoc Intern Med). 2019 Jan;34(1):72-80.

20 Kim ES, Lee HS, Kim SK, Kim EY, Jang BI, Kim KO, et al.; Crohn's and Colitis Association in Daegu-Gyeongbuk (CCAiD). Fecal calprotectin is more accurate than fecal immunochemical test for predicting mucosal healing in quiescent ulcerative colitis: a prospective multicenter study. Scand J Gastroenterol. 2020 Feb;55(2):163-8.

21 D'Haens G, Ferrante M, Vermeire S, Baert F, Noman M, Moortgat L, et al. Fecal calprotectin is a surrogate marker for endoscopic lesions in inflammatory bowel disease. Inflamm Bowel Dis. 2012 Dec;18(12):2218-24.

22 Kristensen V, Klepp P, Cvancarova M, Røseth A, Skar V, Moum B. Prediction of endoscopic disease activity in ulcerative colitis by two different assays for fecal calprotectin. J Crohn's Colitis. 2015 Feb;9(2):164-9.

23 Kristensen V, Røseth A, Ahmad T, Skar V, Moum B. Fecal Calprotectin: A Reliable Predictor of Mucosal Healing after Treatment for Active Ulcerative Colitis. Gastroenterol Res Pract. 2017;2017:2098293.

24 Goll R, Heitmann R, Moe OK, Carlsen K, Florholmen J. Head to head comparison of two commercial fecal calprotectin kits as predictor of Mayo endoscopic sub-score and mucosal TNF expression in ulcerative colitis. PLoS One. 2019 Dec;14(12):e0224895.
25 Theede K, Holck S, Ibsen P, Kallemose T, Nordgaard-Lassen I, Nielsen AM. Fecal Calprotectin Predicts Relapse and Histological Mucosal Healing in Ulcerative Colitis. Inflamm Bowel Dis. 2016 May;22(5):1042-8.

26 Mak WY, Buisson A, Andersen MJ Jr, Lei D, Pekow J, Cohen RD, et al. Fecal Calprotectin in Assessing Endoscopic and Histological Remission in Patients with Ulcerative Colitis. Dig Dis Sci. 2018 May;63(5):1294-301.

27 Zubin G, Peter L. Predicting Endoscopic Crohn's Disease Activity Before and After Induction Therapy in Children: A Comprehensive Assessment of PCDAI, CRP, and Fecal Calprotectin. Inflamm Bowel Dis. 2015 Jun; 21(6):1386-91.

28 Sandborn WJ, Panés J, Zhang H, Yu D, Niezychowski W, Su C. Correlation Between Concentrations of Fecal Calprotectin and Outcomes of Patients With Ulcerative Colitis in a Phase 2 Trial. Gastroenterology. 2016 Jan; 150(1):96-102.

29 Yamaguchi S, Takeuchi Y, Arai K, Fukuda K, Kuroki Y, Asonuma K, et al. Fecal calprotectin is a clinically relevant biomarker of mucosal healing in patients with quiescent ulcerative colitis. J Gastroenterol Hepatol. 2016 Jan;31(1):93-8.

30 Carlsen K, Riis LB, Elsberg H, Maagaard L, Thorkilgaard T, Sørbye SW, et al. The sensitivity of fecal calprotectin in predicting deep remission in ulcerative colitis. Scand J Gastroenterol. 2018 Jun-Jul;53(7):825-30.

31 Kato J, Hiraoka S, Nakarai A, Takashima S, Inokuchi T, Ichinose M. Fecal immunochemical test as a biomarker for inflammatory bowel diseases: can it rival fecal calprotectin? Intest Res. 2016 Jan;14(1):5-14.

32 Vermeire S, Van Assche G, Rutgeerts P. Laboratory markers in IBD: useful, magic, or unnecessary toys? Gut. 2006 Mar;55(3):426-31.

33 Manceau H, Chicha-Cattoir V, Puy H, Peoc'h K. Fecal calprotectin in inflammatory bowel diseases: update and perspectives. Clin Chem Lab Med. 2017 Mar;55(4):474-83.

34 Schoepfer AM, Trummler M, Seeholzer P, Seibold-Schmid B, Seibold F. Discriminating IBD from IBS: comparison of the test performance of fecal markers, blood leukocytes, CRP, and IBD antibodies. Inflamm Bowel Dis. 2008 Jan;14(1):32-9.

35 Baveye S, Elass E, Mazurier J, Spik G, Legrand D. Lactoferrin: a multifunctional glycoprotein involved in the modulation of the inflammatory process. Clin Chem Lab Med. 1999 Mar;37(3):281-6.

36 Mendoza JL, Abreu MT. Biological markers in inflammatory bowel disease: practical consideration for clinicians. Gastroenterol Clin Biol. 2009 Jun;33(Suppl 3):S158-73.

37 Borkowska A, Liberek A, Łuczak G, Jankowska A, Plata-Nazar K, Korzon M, et al. Fecal lactoferrin, a marker of intestinal inflammation in children with inflammatory bowel disease. Acta Biochim Pol. 2015;62(3):541-5.

38 Guerrant RL, Araujo V, Soares E, Kotloff K, Lima AA, Cooper WH, et al. Measurement of fecal lactoferrin as a marker of fecal leukocytes. J Clin Microbiol. 1992 May;30(5):123842 . 
39 Dai C, Jiang M, Sun MJ, Cao Q. Fecal immunochemical test for predicting mucosal healing in ulcerative colitis patients: A systematic review and meta-analysis. J Gastroenterol Hepatol. 2018 May;33(5):990-7.

40 Costa F, Mumolo MG, Bellini M, Romano MR, Ceccarelli L, Arpe P, et al. Role of faeca calprotectin as non-invasive marker of intestinal inflammation. Dig Liver Dis. 2003 Sep; 35(9):642-7.

41 Dolci A, Panteghini M. Comparative study of a new quantitative rapid test with an established ELISA method for faecal calprotectin. Clin Chim Acta. 2012 Jan;413(1-2):350-1.
42 Wassell J, Wallage M, Brewer E; J W. Evaluation of the Quantum Blue ${ }^{\circledR}$ rapid test for faecal calprotectin. Ann Clin Biochem. 2012 Jan; 49(Pt 1):55-8.

43 Kolho KL, Turner D, Veereman-Wauters G, Sladek M, de Ridder L, Shaoul R, et al. Rapid test for fecal calprotectin levels in children with Crohn disease. J Pediatr Gastroenterol Nutr. 2012 Oct;55(4):436-9.

44 Sydora MJ, Sydora BC, Fedorak RN. Validation of a point-of-care desk top device to quantitate fecal calprotectin and distinguish inflammatory bowel disease from irritable bowel syndrome. J Crohn's Colitis. 2012 Mar; 6(2):207-14.
45 Vinding KK, Elsberg H, Thorkilgaard T, Belard E, Pedersen N, Elkjaer M, et al. Fecal Calprotectin Measured By Patients at Home Using Smartphones - A New Clinical Tool in Monitoring Patients with Inflammatory Bowel Disease. Inflamm Bowel Dis. 2016 Feb; 22(2):336-44

46 Elkjaer M, Burisch J, Voxen Hansen V, Deibjerg Kristensen B, Slott Jensen JK, Munkholm P. A new rapid home test for faecal calprotectin in ulcerative colitis. Aliment Pharmacol Ther. 2010 Jan;31(2):323-30. 